\title{
Critical incident in the operating theater: what implication for anaesthesia? An analysis of 155 closed claims (SHAM insurance).
}

\author{
Theissen $A^{1}$, Fuz $F^{2}$, Guerin $\mathrm{JP}^{1}$, Rouquette-Vincenti I ${ }^{1}$, Piriou $\mathrm{V}^{3}$, De Saint-Maurice $\mathrm{G}^{4}$, \\ CAMR (risk analysis and control comitee) of the french society of anaesthesia and intensive care \\ 1 Princess Grace Hospital, Dept of Anaesthesiology \& Intensive Care, Monaco, 2SHAM Insurance, Pole Service, Lyon, France, \\ 3HCL, Dept of Anaesthesiology, Lyon, France, 4Val-de-Grace, military hospital, Dept of Anaesthesiology, Paris, France.
}

\section{Introduction:}

One in 10 hospital inpatients are likely to suffer from an error during their hospital stay. More than sixty percent of critical incidents $(\mathrm{Cl})$ relate to surgery. In two-thirds of cases, $\mathrm{Cl}$ is directly due to a team communication failure.

SHAM insurance is the biggest French provider of medical liability insurance (50\% of the market), insuring $80 \%$ of public and $27 \%$ of private hospitals.

\section{Materials and methods:}

We performed a retrospective study of the closed claims regarding a $\mathrm{Cl}$ which occurred in the operating theatre (or immediately after) before 2010 and settled in a court.

Claims due to obstetrics or nosocomial infection were excluded.

The primary aim of our study was to investigate for the $\mathrm{Cl}$ if anaesthesia was involved.

The secondary aim was to describe the contributing factors of the events (individual factors, communication failure, factors related to the patient ...).

\section{Results:}

The main specialities concerned were orthopaedic surgery $(36 \%)$, visceral surgery $(17 \%)$ and gynaecology (12\%). Consequences were simple $(n=36)$, severe (106) or death (13).

Anaesthesia was involved in 13 cases : wrong intraoperative positioning $(n=3)$, dental injuries (2), post-operative surveillance failure and non-admission to intensive care (2), absence of preoperative visit, wrong evaluation during preoperative consultation, lack of anaesthesia information, medication error, nerve damage after spinal anaesthesia, team medical error.

Surgery remains involved mainly for technical errors (70), lack of information (21), postoperative care or retained foreign objects.

The contributory factor of the event is mainly a cognitive factor $(27 \%)$, a lack of vigilance $(15 \%)$ or surveillance failure $(11 \%)$.

In $22 \%$ of the cases, there is a team communication failure.

\section{Discussion:}

Anaesthesia is rarely involved in the foreground of the $\mathrm{Cl}$.

The closed claims analysis finds a team communication failure. The human factors (individual or related to the team) are almost always at the origin of these claims.

The understanding of the reported claims of this study should help strengthen the quality approach, the simulation program and Team Improvement Programs recently recommended by the HAS (French National Authority for Health). 